\title{
Self-adaptive biosystems through tunable genetic parts and circuits Vittorio Bartoli ${ }^{1}$, Mario di Bernardo ${ }^{1,2,3}$ and Thomas E. Gorochowski ${ }^{2,4}$
}

\begin{abstract}
Biological systems often need to operate in complex environments where conditions can rapidly change. This is possible because of their inherent ability to sense changes and adapt their behavior in response. Here, we detail recent advances in the creation of synthetic genetic parts and circuits whose behaviors can be dynamically tuned through a variety of intracellular and extracellular signals. We show how this capability lays the foundation for implementing control engineering schemes in living cells and allows for the creation of biological systems that are able to self-adapt, ensuring their functionality is maintained in the face of varying environmental and physiological conditions. We end by discussing some of the broader implications of this technology for the safe deployment of synthetic biology.
\end{abstract}

\footnotetext{
Addresses

${ }^{1}$ Department of Engineering Mathematics, University of Bristol, Woodland Road, Bristol, UK

${ }^{2}$ BrisSynBio, University of Bristol, Life Sciences Building, Tyndall Avenue, Bristol, UK

${ }^{3}$ Department of Electrical Engineering and Information Technology, University of Naples Federico II, Via Claudio 21, Napoli, Italy

${ }^{4}$ School of Biological Sciences, University of Bristol, Life Sciences

Building, Tyndall Avenue, Bristol, UK
}

Corresponding author: Gorochowski, Thomas E (thomas.gorochowski@ bristol.ac.uk)

Current Opinion in Systems Biology 2020, 24:78-85

This review comes from a themed issue on Synthetic Biology (2020) Edited by Matteo Barberis and Tom Ellis

For complete overview of the section, please refer the article collection Synthetic Biology (2020)

Available online 14 October 2020

https://doi.org/10.1016/j.coisb.2020.10.006

2452-3100/@ 2020 Elsevier Ltd. All rights reserved.

\section{Keywords}

Tunable, Genetic part, Control, Adaptation, Cybergenetics, Synthetic biology, Systems biology.

\section{Introduction}

A key characteristic of all living organisms is their ability to adapt. From altering metabolism to best utilize shifts in nutrients, to regulating ion transport to maintain cellular homeostasis, adaptive responses are crucial to many aspects of life. To support such adaptive processes, cells have evolved a wide array of sensors able to capture information about their local environment and their internal state. These sensors are connected to cellular circuits that both monitor and modify internal processes with the goal of maintaining specific functionalities (e.g. homeostasis) no matter the perturbations experienced by the cell.

Unlike in nature, engineered biological systems often lack the ability to adapt to changing conditions, making them fragile and causing them to break easily [1-6]. This stems historically from an absence of genetic parts that can be used to dynamically tune the response of a system and the additional burden of implementing control processes on top of a basic functioning system. This view, however, is beginning to change [7-9]. Recent developments in synthetic biology have led to a wide variety of biological parts able to precisely regulate the transcription [10-14] and translation [15-19] of genes in response to diverse intracellular and extracellular signals [20]. Furthermore, the benefits of exploiting control engineering principles to create robust biosystems is also becoming recognized [7-9,21,22]. This stems from a growing need in many applications for reliable and guaranteed functionalities no matter the strain of cell used, or the environment deployed [23].

In this work, we discuss some of the recent advances toward engineering self-adaptive biological systems. We begin by providing an overview of the wide variety of parts now available for sensing and tuning cellular behaviors and show some of the ways these can be used to create adaptive genetic circuits. We then discuss recent steps toward using these circuits to implement closedloop feedback control within living cells to create selfadaptive systems and end by outlining some of the future applications that such capabilities could support.

\section{Tunable genetic parts}

To develop an adaptive system, it is necessary to be able to dynamically alter/tune the input-output relationship of parts within a system. These 'tunable' components come in many different forms, however, conceptually have a common structure (Figure 1a). Each tunable element consists of an input and output, and a further 


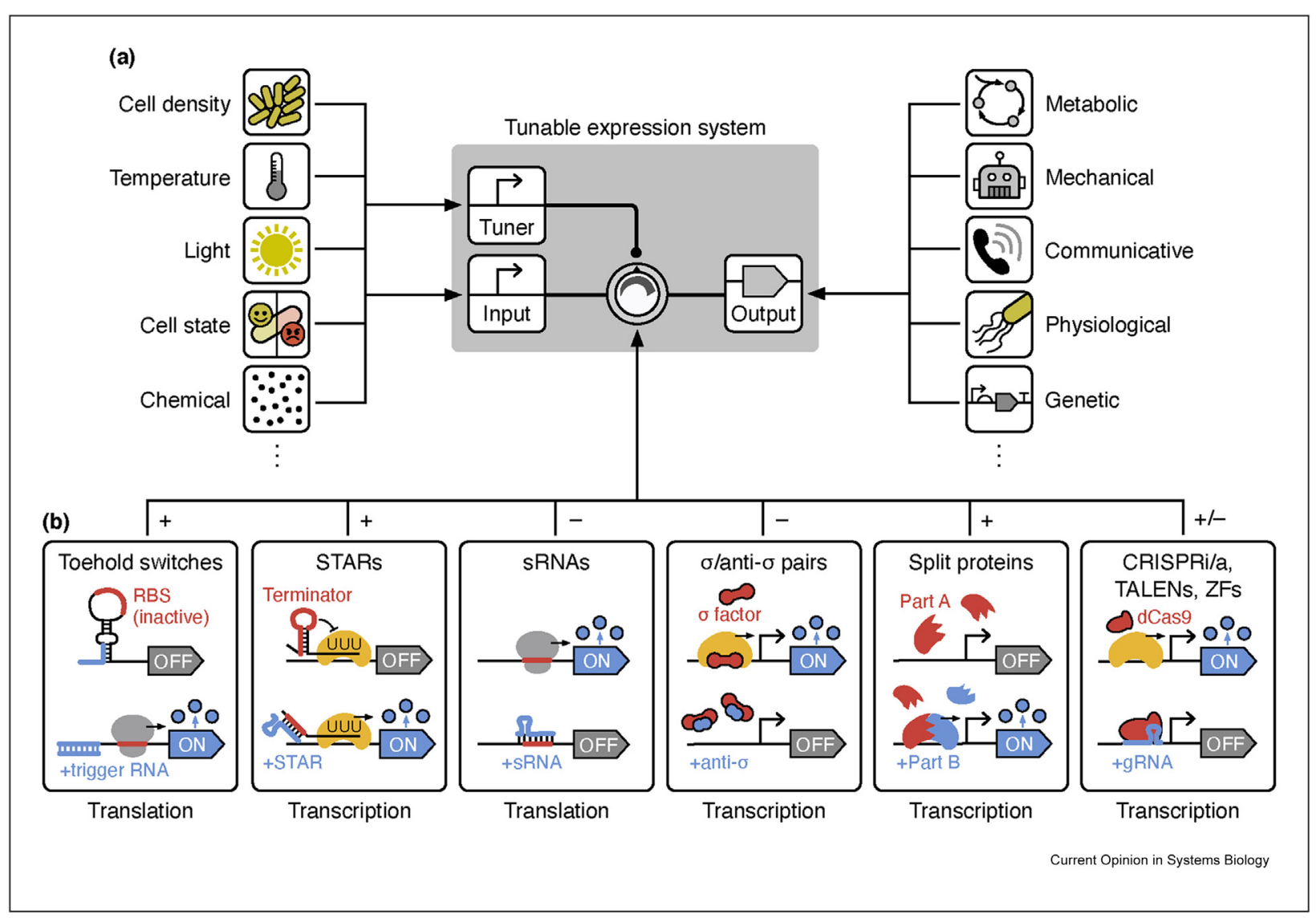

Tunable genetic parts. (a) The schematic of a tunable expression system (TES) where a variety of different inputs and output can be selected. Typically, inputs are transcriptional signals related to environmental or cellular states, and the output is the expression of a gene that influences cellular behavior or acts as an input to another part of a larger circuit. (b) Major regulatory mechanisms that can be used to tune gene expression in a TES. Both active and inactive states shown in addition to whether the tuner will cause activation $(+)$ or repression $(-)$ of the output. The stage in gene expression (i.e. transcription or translation) where regulation takes place is shown below the box for each mechanism. Ribosomes and RNA polymerase (RNAP) shown by light gray and orange shapes without an outline, respectively. For the CRISPRi/a, TALENs and ZFs box a repressive CRISPRi system is shown. This can be modified to be an activator by fusing dCas9 to an activator domain to recruit RNAP to the promoter. In general, the additional blue element would be expressed by the tuner input to modulate expression of the output. RBS, ribosome-binding site; sRNA, small RNA; STAR, small transcription-activating RNA; CRISPRi/a, clustered regularly interspaced short palindromic repeats interference/activation; TALEN, transcription activator-like effector nuclease; ZF, zinc finger; gRNA, guide RNA.

tuner input that is able to alter the input-output relationship in a useful way [24]. Input, output, and tuner signals can take many forms, from gene expression rate to protein phosphorylation state. However, one of the most commonly used is transcriptional activity [3,6,25]. This is captured by the RNA polymerase (RNAP) flux along a strand of DNA and can be directed to particular points by the placement of promoter sequences that control the transcriptional initiation of RNAP. This makes it easy to connect individual parts. You simply make the output promoter of one part the input promoter of another $[10,26]$.

Although there are many ways that the behavior of biological parts can be tuned, the most widespread and easiest to apply is through the control of gene expression. By incorporating additional regulatory elements to modify the rate of transcription and/or translation of an output gene, it is possible to create what we term a tunable expression system (TES) that can vary the amount of output protein produced for a given input transcriptional activity [24]. As gene expression underlies many core cellular behaviors, this approach is a flexible means to control a variety of biological functionalities in a dynamic and tunable way.

The core structure of a TES comprises of promoters acting as signals for the input and tuner, a gene that is expressed as output, and internal regulators that allow the input and tuner promoters to dynamically alter the output protein expression rate (Figure 1a). For the input and tuner promoters, a variety of sensors with 
transcriptional outputs now exist to sense environmental conditions such as chemical concentrations [27] and light [28,29], as well as internal cellular states (e.g. stress responses) [9] and even population level features such as cell density through quorum sensing [30]. Similarly, many output genes exist that enable the control of cellular behaviors from modifying the metabolic state of a cell [31-33] to controlling cell movement [34] and even coordinating cell-to-cell communications [30]. The final component in the TES is the internal regulator used to modulate how transcriptional activity of the input promoter is transformed into an output protein expression rate. To make this relationship a function of the transcriptional activity of the tuner promoter, numerous types of transcriptional and translational regulators can be used (Figure 1b). These include (1) toehold switches where translation rate is controlled through expression of a trigger RNA that is able to disrupt secondary structures around the RBS of the output gene [15,16,24]; (2) small transcription-activating RNAs which interact with transcriptional terminators that are placed in the $5^{\prime}$ untranslated region of a gene and regulate premature RNAP termination [11,12,35]; (3) small RNAs that can be designed to bind the ribosome-binding site for a gene of interest and suppress translation initiation [18]; (4) $\sigma /$ anti- $\sigma$ pairs where the anti- $\sigma$ protein is expressed by the tuner promoter to reduce the expression rate of input promoters driven by the cognate $\sigma$-factor $[36,37]$; (5) split T7 RNAPs where the input and tuner promoters express different halves of the T7 RNAP and the gene of interest is connected to a cognate $\mathrm{T} 7$ promoter [38]; and (6) other programmable transcription factors such as clustered regularly interspaced short palindromic repeats interference/activation (CRISPRi/a) [14], transcription activator-like effector nucleases [39] and zinc fingers [40] that can be expressed by the tuner promoter and interfere or enhance transcription initiation or elongation from the input promoter.

Although it is more common for the input and tuner promoters to be different, recently it has been shown that by using identical promoters to control both regulatory inputs in unison, a more stringent control of protein expression and sharp digital-like transitions between OFF and ON states can be achieved [35,41] (Greco et al. bioRxiv doi: 10.1101/2020.07.04.187500).

It should also be noted that other approaches to tuning gene expression have been developed. For example, two-component systems where phosphorylation rates can be modified by the concentration of specific kinases [42] and CRISPRi systems where the strength of repression is controlled by basepair mismatches in the guide RNA [9]. However, in most cases tuning of such systems requires the physical modification of the encoding DNA making it impossible to dynamically regulate behavior.

\section{Adaptive genetic circuitry}

To implement more complex functionalities, it is often necessary to connect together many genetic parts into a circuit [6]. In other engineering fields such as electronics, specifying the connections between components would generally be sufficient to create a working system. This is due to electronic components having standardized operating ranges to ensure compatibility and reliable functionalities no matter the context they are used in. For example, complementary metal oxidesemiconductor electronic logic gates expect inputs of $0-1.5 \mathrm{~V}$ for an OFF state and 3.5-5 $\mathrm{V}$ for an ON state. In biology, such standardization is difficult because of the diversity of biochemical components used and challenges in engineering them to ensure a common level of response [26,43]. Therefore, rather than imposing constraints on biology that are near impossible to implement, it is instead necessary to work with the diversity present and ensure that components connected have inputs and outputs that are 'matched' to guarantee signals propagate correctly [6,20,44]. Many of the advances in automated genetic circuit design have revolved around ensuring parts perform consistently when used in different ways (e.g. insulating their function from varying genetic context [45,46]) and automating the selection of combinations of parts within a circuit such that their inputs and outputs are optimally matched $[6,26,43]$.

Tunable genetic parts can greatly simplify this process by removing the need to reassemble a circuit if two parts are found to be mismatched when connected. At the cost of additional tuning inputs to a circuit, tunable genetic parts can have their response function dynamically varied after circuit assembly (Figure 2a). This allows parts to be dynamically matched and opens up the possibility of rapidly optimizing overall circuit function without the need to reassemble the underlying DNA (Figure 2b). In addition to simplifying the creation of optimized circuits, the ability to dynamically vary the response dynamics of individual parts is also valuable for systems that must function in highly changeable environments, where shifts might cause physiological changes that impact some or all parts in a circuit $[4,5,47]$.

Beyond the tuning of steady-state response functions, circuits capable of exhibiting dynamic behaviors such as oscillations have also been developed, where characteristics such as the period and amplitude can be varied through diverse inputs to the system. In one such oscillator for Escherichia coli cells, positive and negative feedback loops are implemented using the $\mathrm{P}_{B A D}$ (positive) and $\mathrm{P}_{t a c}$ (negative) systems which can be further regulated using arabinose and isopropyl $\beta$-D-1thiogalactopyranoside, respectively [48]. It was found that increasing the concentration of arabinose caused a 


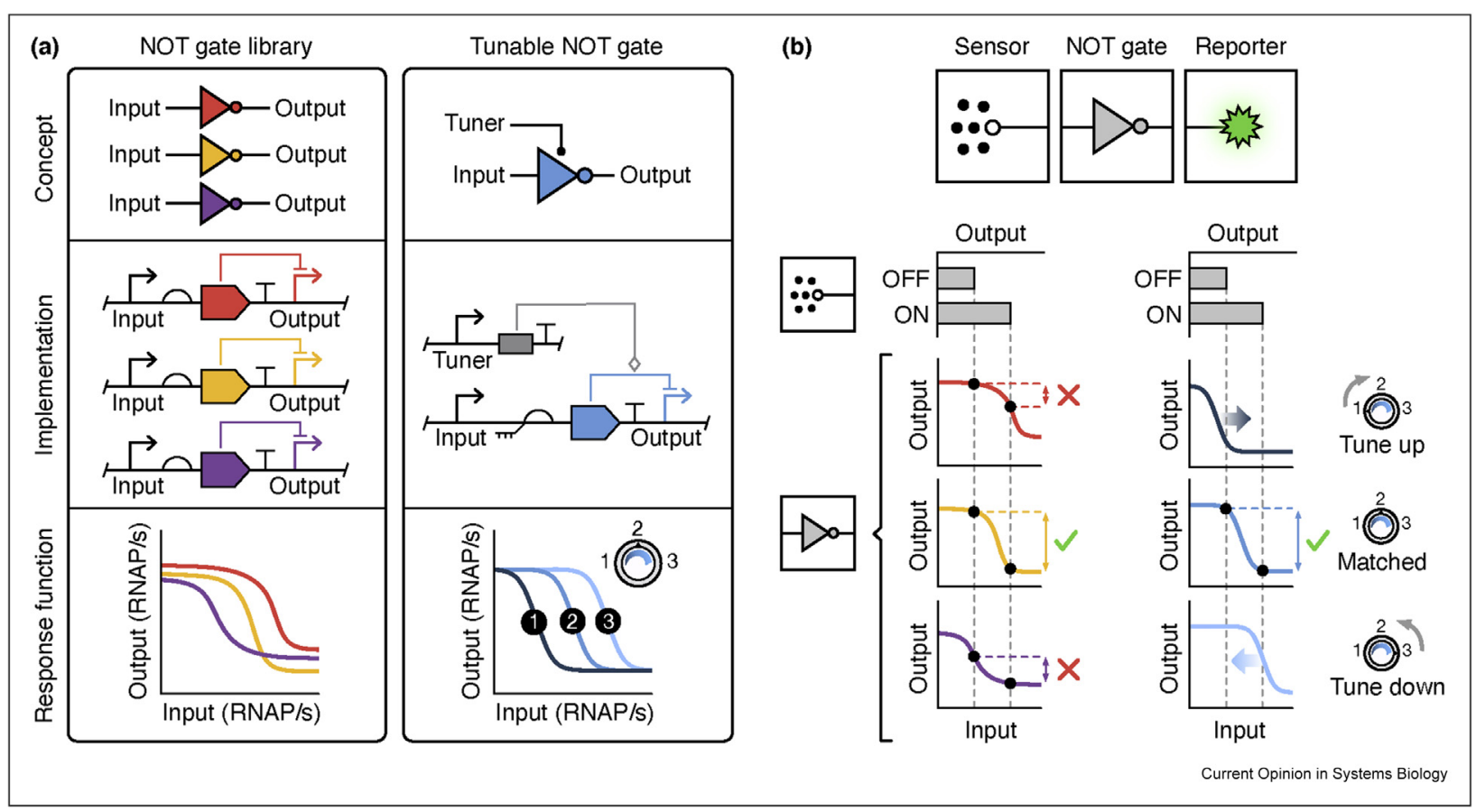

Tunable genetic parts enable the construction of adaptive circuits. (a) Libraries of genetic parts (e.g. NOT gates) are commonly created that cover a range of different behaviors (left box). These differences are shown by the specific response function of each part, which captures the steady-state input-output relationship. For most genetic parts, the response function is fixed and, therefore, physical replacement is necessary if a part is not compatible when used in a system. In contrast, tunable genetic parts (right box) have additional tuner inputs that allow the shape and position of the response function to be dynamically varied as required. (b) The schematic of a simple genetic circuit where a sensor input is inverted to give a desired output reporter (e.g. green fluorescence). For the sensor and NOT gate parts to work effectively, the output of the sensor must 'match' the response function of the NOT gate (dotted gray lines). If the parts are matching, then a large change in the NOT gate output will occur when the sensor switches between OFF and ON states. For standard NOT gates (left column) entire libraries need to be assembled and screened to find a working combination. Furthermore, if the environment changes then too might the behavior of the parts making reassembly necessary. For a tunable NOT gate (right column), the tuner input can be varied until the gate perfectly matches the sensor's outputs. No reassembly is required, allowing the circuit to be dynamically tuned to changing conditions. Genetic circuits shown using Synthetic Biology Open Language (SBOL) Visual notation [73]. RNAP, RNA polymerase.

lengthening of the oscillatory period, whereas increasing an isopropyl $\beta$-D-1-thiogalactopyranoside concentration or temperature led to a shortening of the oscillatory period. Other tunable oscillator circuits have also been developed to allow for control via light [49], to synchronize behaviors across a population of bacteria [50] and to function in mammalian cells [51]. Furthermore, they have been modeled to demonstrate regulatory motifs capable of having their oscillatory amplitude and frequency tuned independently [52].

\section{Toward self-adaptive systems}

A limitation of using tunable genetic parts and circuits is the need for external inputs to be continually provided. A solution to this is to connect the output of a cellular process to the tuner input of the circuit, creating a closed-loop, self-adaptive system. There has been growing interest in the application of closed-loop feedback control in biology and the role that control engineering principles might play in creating robust biosystems [8,21,22,53].
Some simple feedback control schemes have already been implemented in living cells. Many of these focus on the development of dynamic regulatory schemes for metabolism to maximize the yield of desired products $[33,54,55]$. Feedback is created by either using endogenous transcription factors that respond to intermediate metabolites of interest $[31,56]$, or by the design of RNA aptamers able to sense and then actuate gene expression and therefore shift metabolic fluxes in response to changes in metabolite concentrations (Glasscock et al. bioRxiv doi: 10.1101/529180). Related to this, general cellular stress responses have also been used as triggers for feedback control. Specifically, the $\sigma^{32}$ heat-shock response of $E$. coli was found to be rapidly activated when cells were burdened by excessive protein expression [57]. By connecting the endogenous $\mathrm{P}_{h t p G 1} \sigma^{32}$ promoter to a CRISPRi-based feedback control system (Figure 3a), it was shown that protein expression of burdensome synthetic genetic constructs could be dynamically regulated to reduce cellular burden [9]. This both increased the overall protein yield, as there 
Figure 3

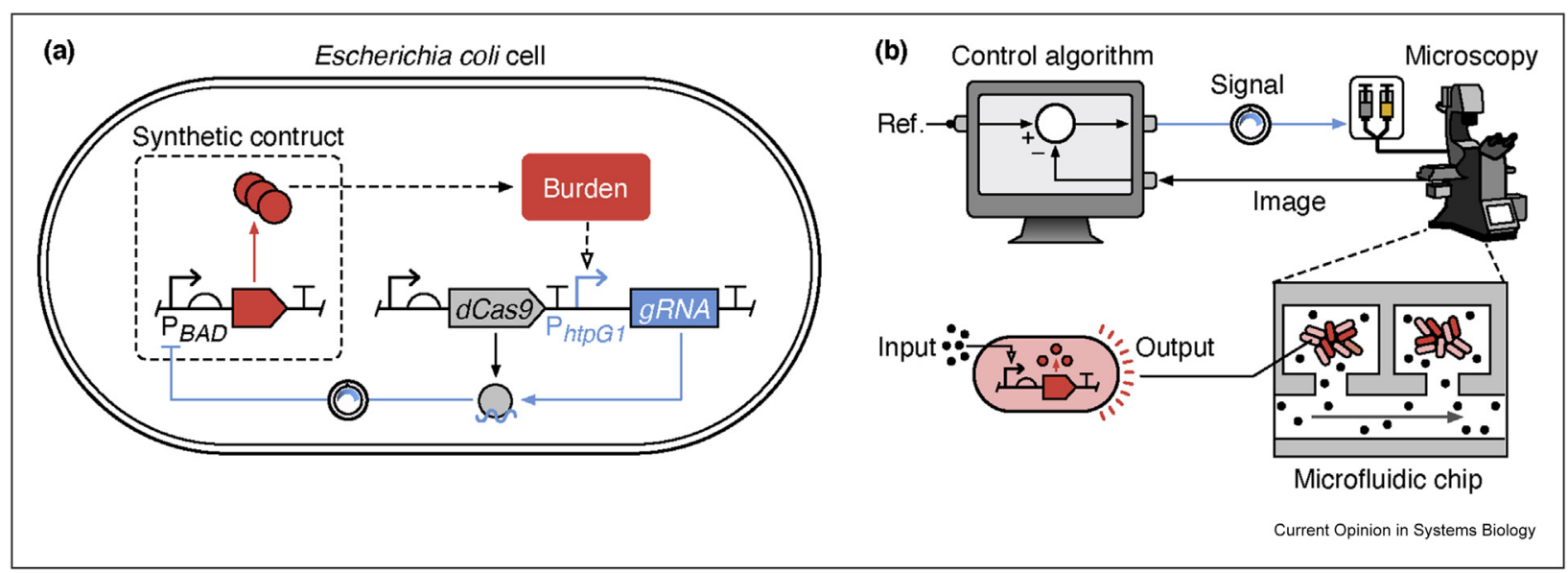

Self-adaptive systems. (a) Embedded burden-based controller. A synthetic construct expresses a burdensome protein. Endogenous cellular processes (dashed arrows) lead to the activation of the $\mathrm{P}_{h t p G 1}$ promoter under high levels of protein expression burden causing expression of a guide RNA (gRNA). This gRNA forms a complex with a constitutively expressed dCas9 protein that then targets the promoter of the synthetic construct, downregulating its expression. The strength of this negative feedback loop is dynamically 'tuned' by the endogenous burden signal and mismatches in the gRNA to the target promoter that reduce the binding affinity of the dCas9:gRNA complex. Panel adapted from the study by Ceroni et al. [9]. (b) Schematic of an external in silico control system. Living cells grow in a microfluidic chip that is continually imaged by a microscope. These images are sent to a computer and analyzed, and an output signal from the cells (e.g. fluorescence) was compared with a desired reference value. A control algorithm assesses this difference and emits a control signal, which actuates syringes and changes the concentration of a signaling molecule provided to the cells. The cells sense this change and alter their gene expression in response. The strength of feedback in this system can be tuned by modulating the control signals produced. The gray arrow in the microfluidic chip represents the flow of nutrients and signaling molecules. gRNA, guide RNA.

was less impact on cellular growth, and the evolutionary stability of the synthetic genetic constructs as there was less selective pressure for mutations. Similar approaches have been implemented using repressor proteins for negative feedback regulation and the $\mathrm{P}_{i b p A B} \sigma^{32}$-promoter as a sensor of burden [58]. Dynamic regulation of protein expression has also been performed in mammalian cells using translation-based negative feedback control [59] and general-purpose gene expression controllers based on quorum sensing [32].

More general feedback control schemes in living cells include the antithetic integral controller motif that uses sequestration mechanisms such as molecular titration to implement an embedded feedback controller [8]. This motif guarantees perfect adaptation, rejecting constant disturbances so that the output of the genetic system of interest initially responds to an external input but then returns to basal levels if the input persists [21].

Molecular titration has also been shown to be an effective mechanism to implement 'comparator' devices able to produce an output function capturing the mismatch between the levels of two different inputs - an essential component of any biomolecular controller [36,60]. Implementations of more sophisticated control strategies have also been recently presented, such as the biomolecular Proportional-Integral-Derivative (PID) controller presented in the study by Chevalier et al. [61]. As the complexity of biomolecular control designs increases, to successfully implement the control function, parts must be finely tuned to guarantee the right balance between the sensing and actuating functions of a circuit [62]. The use of tunable parts could open the way to the development of adaptive biomolecular controllers able to self-tune themselves to guarantee the robust execution of the control task they are assigned, being able to function in the presence of perturbations, cellto-cell variability, and so on. This might be even more crucial when the control functions themselves are spread among different populations in a microbial consortium as recently suggested by Fiore et al. [63].

Beyond simple feedback motifs, it can be difficult to implement complex control algorithms using biochemical components because the feedback strengths and dynamics required may be difficult to match to available parts. Therefore, an intermediate step is sometimes taken whereby a computer is used to implement controller logic within the feedback loop and create what is termed a cybergenetic system [22] (Figure 3b). Cybergenetic systems often rely on single-cell microcopy platforms and microfluidics to image engineered cells whose current state is displayed via fluorescent reporter proteins and use chemical inducers $[64,65]$ or light $[28,29,66,67]$ as inputs to perturb the cells states in a predefined way 
(i.e. the cells are engineered to sense and update their state in response to a stimulus). The computerbased controller runs in real-time analyzing microscopy images to extract the current states of cells and then immediately computes a control action, which is then administered by varying the chemical concentrations or light that the cells are exposed to. Such systems have been shown capable of controlling both population $[8,64,66,68]$ and single-cell behaviors [67]. Moreover, toolkits have emerged to simplify their creation by handling image analysis, tracking, and calculation of control actions (Pedone et al. bioRxiv doi: 10.1101/2020.06.25.171751). The major advantage of this hybrid approach is that the computer controllers are cell-agnostic, allowing them to be used with any biosystem that has the same types of control inputs and observable outputs.

\section{Conclusions}

The creation of self-adaptive biosystems that can function in the face of varying and uncertain environments will be a crucial step for the safe deployment of synthetic biology into everyday life. Recent advances in biological control engineering provide the theoretical foundations necessary to design such systems, and, as we have shown, tunable genetic parts and circuits can support their physical implementation [24]. While the self-adaptive systems built to date have mostly been small-scale proof-of concepts, it is clear that the ability to synthesize and assemble entire genomes is in reach $[69,70]$. Demonstrating the value of integrating tunable parts and circuits within these cellular systems will be crucial to moving beyond the mere recoding of existing genomic information and toward the creation of synthetic cells built from the ground up to reliably implement novel functionalities. Furthermore, they will support the scale-up of these systems by enabling us to move beyond single cells and toward the engineering of collective behaviors of vast populations of cells [71] or even entire synthetic ecologies [72].

\section{Author contributions}

All authors contributed to the writing and editing. T.E.G. and V.B. produced the figures.

\section{Conflict of interest statement}

Nothing declared.

\section{Acknowledgements}

This work was supported by BrisSynBio, a BBSRC/EPSRC Synthetic Biology Research Centre grant BB/L01386X/1 (M.d.B., T.E.G.), EPSRC/ BBSRC Centre for Doctoral Training in Synthetic Biology grant EP/ L016494/1 (V.B.), the EU Horizon 2020 research project COSY-BIO grant 766840 (M.d.B), and a Royal Society University Research Fellowship grant UF160357 (T.E.G.). This study did not involve any underlying data.

\section{References}

Papers of particular interest, published within the period of review, have been highlighted as:
- of special interest

- of outstanding interest

1. Fernandez-Rodriguez J, Yang L, Gorochowski TE, Gordon DB, Voigt CA: Memory and combinatorial logic based on DNA inversions: dynamics and evolutionary stability. ACS Synth Biol 2015, 4:1361-1372.

2. Sleight SC, Bartley BA, Lieviant JA, Sauro HM: Designing and engineering evolutionary robust genetic circuits. J Biol Eng 2010, 4:12.

3. Gorochowski TE, Espah Borujeni A, Park Y, Nielsen AA, Zhang J, Der BS, Gordon DB, Voigt CA: Genetic circuit characterization and debugging using RNA-seq. Mol Syst Biol 2017, 13:952.

4. Gorochowski TE, van den Berg E, Kerkman R, Roubos JA, Bovenberg RAL: Using synthetic biological parts and microbioreactors to explore the protein expression characteristics of Escherichia coli. ACS Synth Biol 2014, 3:129-139.

5. Moser F, Broers NJ, Hartmans S, Tamsir A, Kerkman R, Roubos JA, Bovenberg R, Voigt CA: Genetic circuit performance under conditions relevant for industrial bioreactors. ACS Synth Biol 2012, 1:555-564.

6. Brophy JAN, Voigt CA: Principles of genetic circuit design. Nat Methods 2014, 11:508.

7. Segall-Shapiro TH, Sontag ED, Voigt CA: Engineered promoters enable constant gene expression at any copy number in bacteria. Nat Biotechnol 2018, 36:352.

8. Aoki SK, Lillacci G, Gupta A, Baumschlager A, Schweingruber D,

-• Khammash M: A universal biomolecular integral feedback controller for robust perfect adaptation. Nature 2019, 570: 533-537.

Details the discovery of a universal biochemical controller topology for achieving integral feedback and perfect adaptation. This regulatory motif is further implemented using $\sigma / a n t i-\sigma$ pairs and used to robustly control the growth rate of Escherichia coli cells. Other tunable parts that rely on sequestering of molecular species could offer additional means to implement similar control systems.

9. Ceroni F, Boo A, Furini S, Gorochowski TE, Borkowski O, Ladak YN, - Awan AR, Gilbert C, Stan G-B, Ellis T: Burden-driven feedback control of gene expression. Nat Methods 2018, 15:387-393.

Details the development of a feedback control system to automatically regulate the burden experienced by cells expressing a synthetic genetic construct. The major contribution is the detailed analysis of endogenous cellular responses to find a suitable burden-responsive promoter and the use of a tunable CRISPRi feedback regulatory mechanism that enables any synthetic construct to be targeted. It is shown that this system can significantly improve the yield of protein products, as well as the evolutionary stability of genetic constructs.

10. Stanton BC, Nielsen AAK, Tamsir A, Clancy K, Peterson T, Voigt CA: Genomic mining of prokaryotic repressors for orthogonal logic gates. Nat Chem Biol 2014, 10:99-105.

11. Chappell J, Westbrook A, Verosloff M, Lucks JB: Computational design of small transcription activating RNAs for versatile and dynamic gene regulation. Nat Commun 2017, 8:1051.

12. Chappell J, Takahashi MK, Lucks JB: Creating small transcription activating RNAs. Nat Chem Biol 2015, 11:214-220.

13. Kim H, Bojar D, Fussenegger M: A CRISPR/Cas9-based central processing unit to program complex logic computation in human cells. Proc Natl Acad Sci U S A 2019, 116:7214.

14. Gilbert LA, Horlbeck MA, Adamson B, Villalta JE, Chen Y, Whitehead EH, Guimaraes C, Panning B, Ploegh HL, Bassik MC, et al.: Genome-scale CRISPR-mediated control of gene repression and activation. Cell 2014, 159:647-661.

15. Green AA, Silver PA, Collins JJ, Yin P: Toehold switches: denovo-designed regulators of gene expression. Cell 2014, 159 : 925-939.

16. Green AA, Kim J, Ma D, Silver PA, Collins JJ, Yin P: Complex cellular logic computation using ribocomputing devices. Nature 2017, 548:117.

17. Gorochowski TE, Chelysheva I, Eriksen M, Nair P, Pedersen S, Ignatova Z: Absolute quantification of translational regulation 
and burden using combined sequencing approaches. Mol Syst Biol 2019, 15, e8719.

18. Kelly CL, Harris AWK, Steel H, Hancock EJ, Heap JT, Papachristodoulou A: Synthetic negative feedback circuits using engineered small RNAs. Nucleic Acids Res 2018, 46: 9875-9889.

19. Soper T, Mandin P, Majdalani N, Gottesman S, Woodson SA: Positive regulation by small RNAs and the role of Hfq. Proc Natl Acad Sci U S A 2010, 107:9602.

20. Greco FV, Tarnowski MJ, Gorochowski TE: Living computers powered by biochemistry. Biochemist 2019, 41:14-18.

21. Briat C, Gupta A, Khammash M: Antithetic integral feedback ensures robust perfect adaptation in noisy biomolecular networks. Cell Syst 2016, 2:15-26.

22. Khammash M, Di Bernardo M, Di Bernardo D: Cybergenetics:

- theory and methods for genetic control system. In 2019 IEEE 58th Conference on Decision and Control (CDC); 2019:916-926.

A clear introduction to the emerging field of cybergenetics covering the key theory and experimental methods that make these types of system possible.

23. Haringa C, Tang W, Wang G, Deshmukh AT, van Winden WA, Chu J, van Gulik WM, Heijnen JJ, Mudde RF, Noorman HJ: Computational fluid dynamics simulation of an industrial P. chrysogenum fermentation with a coupled 9-pool metabolic model: towards rational scale-down and design optimization. Chem Eng Sci 2018, 175:12-24.

24. Bartoli V, Meaker GA, di Bernardo M, Gorochowski TE: Tunable

-• genetic devices through simultaneous control of transcription and translation. Nat Commun 2020, 11:2095.

The first time a generalized tunable expression system (TES) is developed. The TES is used to create tunable logic gates (NOT and NOR) that are more compatible than existing parts, and some key biomolecular design constraints are explored for future development of tunable part and systems.

25. Canton B, Labno A, Endy D: Refinement and standardization of synthetic biological parts and devices. Nat Biotechnol 2008 26:787.

26. Nielsen AAK, Der BS, Shin J, Vaidyanathan P, Paralanov V, Strychalski EA, Ross D, Densmore D, Voigt CA: Genetic circuit design automation. Science 2016, 352:aac7341.

27. Meyer AJ, Segall-Shapiro TH, Glassey E, Zhang J, Voigt CA: Escherichia coli "Marionette" strains with 12 highly optimized small-molecule sensors. Nat Chem Biol 2019, 15:196-204.

28. Baumschlager A, Aoki SK, Khammash M: Dynamic blue lightinducible T7 RNA polymerases (Opto-T7RNAPs) for precise spatiotemporal gene expression control. ACS Synth Biol 2017 , 6:2157-2167.

29. Castillo-Hair SM, Baerman EA, Fujita M, Igoshin OA, Tabor JJ: Optogenetic control of Bacillus subtilis gene expression. Nat Commun 2019, 10:3099.

30. Scott SR, Hasty J: Quorum sensing communication modules for microbial consortia. ACS Synth Biol 2016, 5:969-977.

31. Doong SJ, Gupta A, Prather KLJ: Layered dynamic regulation

- for improving metabolic pathway productivity in Escherichia coli. Proc Natl Acad Sci U S A 2018, 115:2964.

Employs the novel use of two orthogonal and tunable regulators to dynamically control and optimize several aspects of cellular metabolism for D-glucaric acid production. Highlights the value of tunable regulation for metabolic applications and the highest reported titers of glucaric acid in Escherichia coli cells.

32. Gupta A, Reizman IMB, Reisch CR, Prather KLJ: Dynamic regulation of metabolic flux in engineered bacteria using a pathway-independent quorum-sensing circuit. Nat Biotechno 2017, 35:273.

33. Moser F, Espah Borujeni A, Ghodasara AN, Cameron E, Park Y Voigt CA: Dynamic control of endogenous metabolism with combinatorial logic circuits. Mol Syst Biol 2018, 14, e8605.

34. Blair KM, Turner L, Winkelman JT, Berg HC, Kearns DB: A molecular clutch disables flagella in the Bacillus subtilis biofilm. Science 2008, 320:1636-1638.
35. Westbrook AM, Lucks JB: Achieving large dynamic range control of gene expression with a compact RNA transcription-translation regulator. Nucleic Acids Res 2017, 45:5614-5624.

36. Annunziata F, Matyjaszkiewicz A, Fiore G, Grierson CS, Marucci L, di Bernardo M, Savery NJ: An orthogonal multi-input integration system to control gene expression in Escherichia coli. ACS Synth Biol 2017, 6:1816-1824.

37. Rhodius VA, Segall-Shapiro TH, Sharon BD, Ghodasara A Orlova E, Tabakh H, Burkhardt DH, Clancy K, Peterson TC, Gross CA, et al.: Design of orthogonal genetic switches based on a crosstalk map of $\sigma \mathbf{s}$, anti- $\sigma \mathbf{s}$, and promoters. Mol Syst Biol 2013, 9:702.

38. Segall-Shapiro TH, Meyer AJ, Ellington AD, Sontag ED, Voigt CA: A 'resource allocator' for transcription based on a highly fragmented T7 RNA polymerase. Mol Syst Biol 2014, 10:742.

39. Moore R, Chandrahas A, Bleris L: Transcription activator-like effectors: a toolkit for synthetic biology. ACS Synth Biol 2014 3:708-716.

40. Khalil AS, Lu TK, Bashor CJ, Ramirez CL, Pyenson NC, Joung JK, Collins JJ: A synthetic biology framework for programming eukaryotic transcription functions. Cell 2012, 150 647-658.

41. Calles B, Goñi-Moreno Á, de Lorenzo V: Digitalizing heterologous gene expression in Gram-negative bacteria with a portable ON/OFF module. Mol Syst Biol 2019, 15:e8777.

42. Schmidl SR, Sheth RU, Wu A, Tabor JJ: Refactoring and optimization of light-switchable Escherichia coli two-component systems. ACS Synth Biol 2014, 3:820-831.

43. Vaidyanathan P, Der BS, Bhatia S, Roehner N, Silva R, Voigt CA Densmore D: A framework for genetic logic synthesis. Proc IEEE 2015, 103:2196-2207.

44. Grozinger L, Amos M, Gorochowski TE, Carbonell P, Oyarzún DA, Stoof R, Fellermann H, Zuliani P, Tas H, GoñiMoreno A: Pathways to cellular supremacy in biocomputing. Nat Commun 2019, 10:5250.

45. Qi L, Haurwitz RE, Shao W, Doudna JA, Arkin AP: RNA processing enables predictable programming of gene expression. Nat Biotechnol 2012, 30:1002.

46. Lou C, Stanton B, Chen Y-J, Munsky B, Voigt CA: Ribozymebased insulator parts buffer synthetic circuits from genetic context. Nat Biotechnol 2012, 30:1137.

47. Wohlgemuth SE, Gorochowski TE, Roubos JA: Translational sensitivity of the Escherichia coli genome to fluctuating tRNA availability. Nucleic Acids Res 2013, 41:8021-8033.

48. Stricker J, Cookson S, Bennett MR, Mather WH, Tsimring LS, Hasty J: A fast, robust and tunable synthetic gene oscillator Nature 2008, 456:516.

49. Mahajan T, Rai K: A novel optogenetically tunable frequency modulating oscillator. PloS One 2018, 13, e0183242.

50. Ryback BM, Odoni DI, van Heck RG, van Nuland $Y$, Hesselman MC, Martins dos Santos VA, van Passel MW, Hugenholtz F: Design and analysis of a tunable synchronized oscillator. J Biol Eng 2013, 7:26.

51. Tigges M, Marquez-Lago TT, Stelling J, Fussenegger M: A tunable synthetic mammalian oscillator. Nature 2009, 457: 309-312.

52. Tomazou M, Barahona M, Polizzi KM, Stan G-B: Computational

- Re-design of synthetic genetic oscillators for independent amplitude and frequency modulation. Cell Syst 2018, 6: 508-520.e5.

Theoretical study demonstrating how genetic oscillators can be redesigned to enable the independent tuning of amplitude and frequency. The major contribution is the discovery of the important role that enzymatic degradation can play in allowing a decoupling of internal processes, and thus tuning of system dynamics.

53. Del Vecchio D, Dy AJ, Qian Y: Control theory meets synthetic biology. J R Soc Interface 2016, 13:20160380. 
54. Zhang J, Jensen MK, Keasling JD: Development of biosensors and their application in metabolic engineering. Synth Biol • Synth Biomol 2015, 28:1-8.

55. Tan SZ, Prather KL: Dynamic pathway regulation: recent advances and methods of construction. Mech Biol Energy 2017, 41:28-35.

56. Chou HH, Keasling JD: Programming adaptive control to evolve increased metabolite production. Nat Commun 2013, 4 2595.

57. Ceroni F, Algar R, Stan G-B, Ellis T: Quantifying cellular capacity identifies gene expression designs with reduced burden. Nat Methods 2015, 12:415-418.

58. Dragosits M, Nicklas D, Tagkopoulos I: A synthetic biology approach to self-regulatory recombinant protein production in Escherichia coli. J Biol Eng 2012, 6:2.

59. Stapleton JA, Endo K, Fujita Y, Hayashi K, Takinoue M, Saito H Inoue $\mathrm{T}$ : Feedback control of protein expression in mammalian cells by tunable synthetic translational inhibition. ACS Synth Biol 2012, 1:83-88.

60. Cuba Samaniego C, Giordano G, Kim J, Blanchini F, Franco E: Molecular titration promotes oscillations and bistability in minimal network models with monomeric regulators. ACS Synth Biol 2016, 5:321-333.

61. Chevalier M, Gómez-Schiavon M, Ng AH, El-Samad H: Design and analysis of a proportional-integral-derivative controller with biological molecules. Cell Syst 2019, 9. 338-353.e10.

62. Filo M, Khammash M: Optimal parameter tuning of feedback controllers with application to biomolecular antithetic integral control. In 2019 IEEE 58th Conference on Decision and Control (CDC); 2019:951-957.

63. Fiore G, Matyjaszkiewicz A, Annunziata F, Grierson C, Savery NJ, Marucci L, di Bernardo M: In-silico analysis and implementation of a multicellular feedback control strategy in a synthetic bacterial consortium. ACS Synth Biol 2017, 6: 507-517.

64. Fiore G, Perrino G, di Bernardo M, di Bernardo D: Vivo real-time

- control of gene expression: a comparative analysis of feedback control strategies in yeast. ACS Synth Biol 2016, 5: 154-162.

Demonstration of the ability to combine single-cell imaging of yeast cells in microfluidics with real-time feedback control of chemical inducers to robustly regulate gene expression. A number of control algorithms are tested, with the performance of each varying for different tasks.

65. Postiglione L, Napolitano S, Pedone E, Rocca DL, Aulicino F, Santorelli M, Tumaini B, Marucci L, di Bernardo D: Regulation of gene expression and signaling pathway activity in mammalian cells by automated microfluidics feedback control. ACS Synth Biol 2018, 7:2558-2565.

66. Milias-Argeitis A, Rullan M, Aoki SK, Buchmann P, Khammash M:

-. Automated optogenetic feedback control for precise and robust regulation of gene expression and cell growth. Nat Commun 2016, 7:12546.

Describes the development of an automated system to allow for reliable long-term optogenetic control-based experiments. Combines tunable expression systems based on two-component light sensors and supporting hardware for continuous culture, automated sampling and analysis. Demonstrates the value of fully integrated and self-adaptive systems.

67. Rullan M, Benzinger D, Schmidt GW, Milias-Argeitis A, Khammash M: An optogenetic platform for real-time, singlecell interrogation of stochastic transcriptional regulation. $\mathrm{Mol}$ Cell 2018, 70:745-756.e6.

68. Pedone E, Postiglione L, Aulicino F, Rocca DL, Montes-Olivas S, - Khazim M, di Bernardo D, Pia Cosma M, Marucci L: A tunable dual-input system for on-demand dynamic gene expression regulation. Nat Commun 2019, 10:4481.

Describes a new multi-level system for controlling gene expression in mammalian cells. By combining transcriptional control with posttranslational destabilization, it is possible to rapidly modulate protein expression level. The benefits of these properties for control applications are also shown.

69. Fredens J, Wang K, de la Torre D, Funke LFH, Robertson WE, Christova Y, Chia T, Schmied WH, Dunkelmann DL, Beránek V, et al.: Total synthesis of Escherichia coli with a recoded genome. Nature 2019, 569:514-518.

70. Richardson SM, Mitchell LA, Stracquadanio G, Yang K, Dymond JS, DiCarlo JE, Lee D, Huang CLV, Chandrasegaran S, Cai $Y$, et al.: Design of a synthetic yeast genome. Science $2017,355: 1040$.

71. Karkaria BD, Treloar NJ, Barnes CP, Fedorec AJH: From microbial communities to distributed computing systems. Front Bioeng Biotechnol 2020, 8:834.

72. Gorochowski TE, Hauert S, Kreft J-U, Marucci L, Stillman NR, Tang T-YD, Bandiera L, Bartoli V, Dixon DOR, Fedorec AJH, et al.: Toward engineering biosystems with emergent collective functions. Front Bioeng Biotechnol 2020, 8:705.

73. Beal J, Nguyen T, Gorochowski TE, Goñi-Moreno A, ScottBrown J, McLaughlin JA, Madsen C, Aleritsch B, Bartley B, Bhakta $S$, et al.: Communicating structure and function in synthetic biology diagrams. ACS Synth Biol 2019, 8: $1818-1825$ 